\title{
Elementos auxiliares de construcción en la arquitectura gótica. El pilar «major» de la catedral de Tortosa
}

\section{Auxiliar elements of construction in gothic architecture. The "pilar major" of the cathedral of Tortosa}

J. Lluis i Ginovart $^{(*)}$, A. Costa-Jover ${ }^{(*)}$, G. Fortuny i Anguera ${ }^{(*)}$

RESUMEN

La construcción gótica no habría sido posible sin la utilización de medios auxiliares que soportaran los empujes de la estructura durante el proceso de montaje. En el caso de la catedral gótica de Tortosa, los libros de obra hacen referencia a un gran pilar utilizado durante la construcción del ábside, al que se refieren como pilar major.

La lógica constructiva, junto con el conocimiento sobre las fases de ejecución, han permitido deducir el funcionamiento y ubicación de este elemento. De manera complementaria, se ha verificado gráficamente que el equilibrio del ábside no es posible antes del cierre del presbiterio sin un elemento de contrarresto.

El estudio del subsuelo por medio de un georadar ha permitido identificar una masa sólida situada en el centro del ábside, que coincide con la ubicación del pilar major y que habría servido como base de cimentación para su construcción.

Palabras clave: Catedral; georadar; gótico; medios auxiliares.

\section{ABSTRACT}

Gothic construction wouldn't have been possible without the use of auxiliary resources which could handle the efforts of the structure during the raising process. In the cathedral of Tortosa case, the 'libros de obra' refers to a big pillar used during the building of the apse, which is named 'pilar major'.

The logic of the construction, and also the knowledge about the building phases, has let to deduce the role and the location of this element. Complementarily, it have been verified by graphical methods that the equilibrium of the apse is not possible before the presbitery is closed, without an element of buttressing.

The study of the subsoil by means of a georadar has let to identify a solid mass on the center of the apse, where the 'pilar major' would be situated. It probably could be the foundation for its construction.

Keywords: Cathedral; georadar; gothic; auxiliary resources.

(*) Universitat Rovira i Virgili. Reus (España).

Persona de contacto/Corresponding author: josep.lluisg@urv.cat (J. Lluís i Ginovart)

Cómo citar este artículo/Citation: Lluis i Ginovart, J., Costa-Jover, A., Fortuny i Anguera, G. (2015). Elementos auxiliares de construcción en la arquitectura gótica. El pilar «major» de la catedral de Tortosa. Informes de la Construcción, 67(537): eo57, doi: http://dx.doi.org/10.3989/ic.13.059.

Licencia / License: Salvo indicación contraria, todos los contenidos de la edición electrónica de Informes de la Construcción se distribuyen bajo una licencia de uso y distribución Creative Commons Reconocimiento no Comercial 3.o. España (cc-by-nc). 


\section{INTRODUCCIÓN}

El ábside de la catedral de Tortosa fue construido entre (13741441). En los libros de fábrica (Llibres d'Obra - Ll.o.) del Archivo Capitular de Tortosa (ACTo) aparece la construcción de un pilar major. (Figura 1). Victoria Almuni (2007) lo sitúa en 1428 , en plena construcción del sector central del presbiterio (1). Inicialmente se planteó la hipótesis de una construcción auxiliar, como elemento de soporte para la colocación de la clave del presbiterio, dadas sus dimensiones; 2,32 metros de diámetro y un peso de más 8 Tn. La colocación de ésta puede considerarse como el mayor alarde técnico en la construcción de la catedral. La clave representa la Coronación de la Virgen María tras su Ascensión a los Cielos, se eleva mediante un ceremonial público, la festividad de la Asunción (2) (Figura 2). Josep Lluis (2011) plantea la tesis de una construcción auxiliar, que luego será desmontada, pero como elemento esencial para el equilibrio en la construcción del deambulatorio (3). Por otra parte Artur Llunart y Josep Lluis (2013) (4) realizan una primera hipótesis constructiva de los sistemas de andamiaje en la construcción del ábside, utilizando el pilar como elemento de apeo de éstos.

La construcción de las bóvedas es consecuencia de unas técnicas constructivas, cuyo principio está basado en la utilización de elementos y estructuras auxiliares en las fases intermedias de la ejecución de la fábrica. Las noticias de Hernán Ruiz el Joven, (c.1514-1569) y sus técnicas para la construcción de bóvedas, recogidas por Simón García (f.1681), en el Compendio de arquitectura (1681), (5) son una de las escasas las fuentes directas del mundo gótico. Como lo son también las noticias delos peritajes para la secuencia constructiva de bóvedas de Enrique Egas (1455-1534) en 1532 y de Francisco de Colonia (1470-1542) en 1536, para la construcción de las bóvedas de la Catedral de Segovia (6) (7). Los cimbrados a gran altura fueron utilizados por los restauradores españoles del siglo XIX; Juan de Madrazo (1874) y Demetrio de los Rios (1879) en la catedral de León (8), Fernández Casanova en la de Sevilla (1882-1888) (9), Repullés y Vargas en San Vicente de Ávila (1889), o Vicente Lampérez en la catedral de Cuenca (1888-1889) (10).

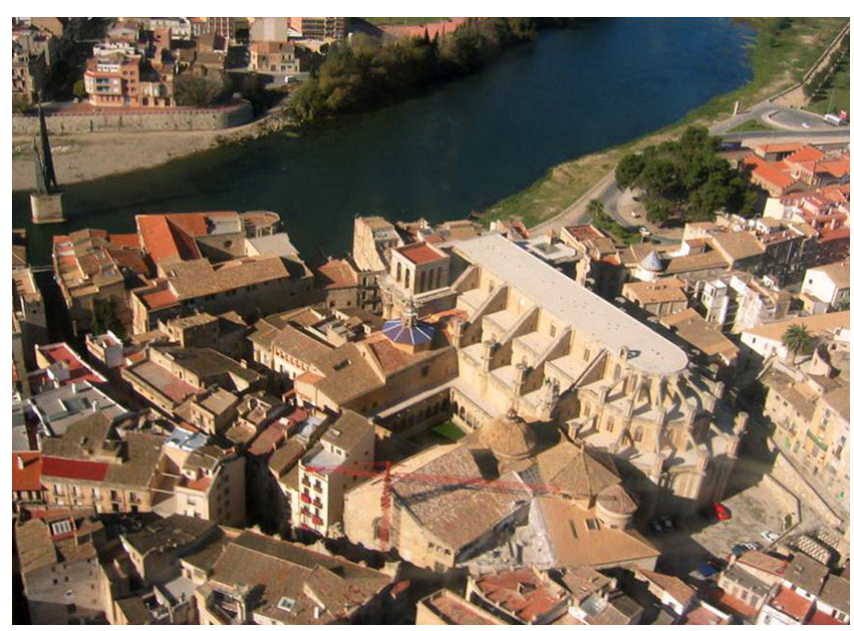

Figura 1. Catedral de Santa María de Tortosa.

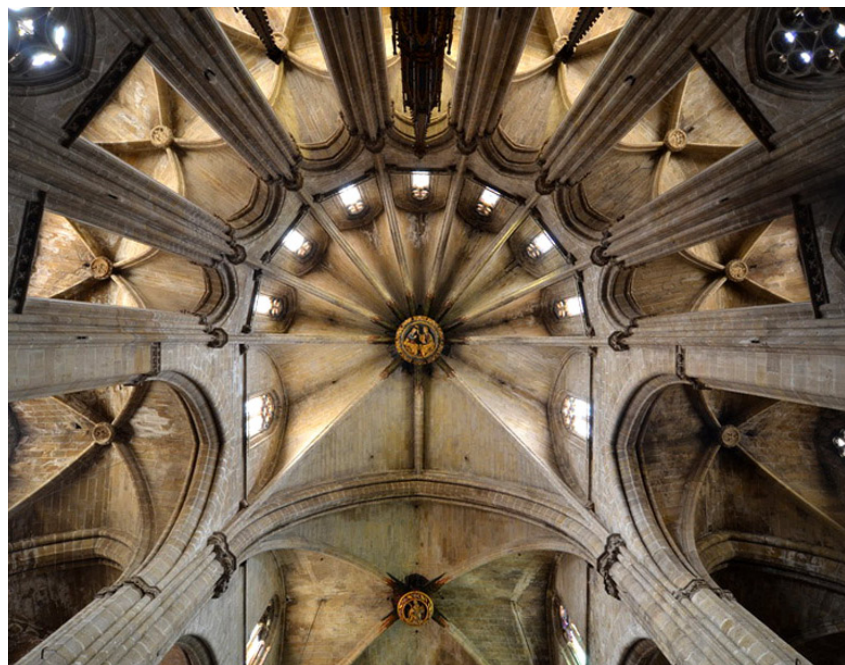

Figura 2. Presbiterio con la Clave mayor. La Coronación de la Virgen María (1439).

\section{OBJETIVO Y MÉTODO}

Los estudios de Alumuni (2007), Lluis (2011), Llunart; Lluis (2013), partieron de una ubicación hipotética, tanto del pilar major, como de la catedral románica, a la que la catedral gótica sustituyó. El estudió tiene como objetivo plantear los resultados de los análisis geofísicos del subsuelo de la catedral, tanto en lo referente a la ubicación geográfica de este elemento auxiliar, así como de la función de este pilar desde el punto de vista estructural.

Los análisis geofísicos son similares a los realizados en las catedrales de Girona (11) y Tarragona (12), permitiendo localizar las anomalías en el subsuelo, y pudiendo hipotetizar sobre la ubicación topográfica del pilar auxiliar. Una vez situado éste, se realiza el estudio de la sección de las fases constructivas, sometidas a un análisis límite, similares a los realizados en la catedral de Segovia (13). Todo ello va a permitir complementar las tesis establecidas por los estudios precedentes, apoyados en una lectura transversal sobre las fuentes historiográficas (Figura 3).

\section{EL CINTURÓN DE LAS CAPILLAS RADIALES, LA APARICIÓN DEL PILAR MAJOR}

En el inicio de la obra el maestro, solicita para la traza del ábside la construcción de una cana, (Ll.o.2 1345-1347, f.36 v) ${ }^{1}$ (14). El patrón básico de las medidas, de los Llibres d'Obra (ACTo), es la cana de 8 palmos. La Cana de Tortosa utilizada en la catedral, tiene $1.858 \mathrm{~cm}$ y el palmo de $23,23 \mathrm{~cm}^{2}$. El proceso de construcción de las capillas radiales se realiza a modo de cinturón alrededor de la catedral románica. Las bóvedas son cubiertas de forma correlativa y secuencial, desde el sector del evangelio al de la epístola entre 1377 y 1424. Las capillas son de planta cuadrada, cubierta con bóveda de crucería. La distancia entre pilares es de 24 palmos, 3 canas de Tortosa. La luz de las bóvedas es de 21 palmos, generando respectivamente una base metrológica de $8 \times 8$ y $7 \times 7$, sobre una estructura de base 9.

\footnotetext{
Item fiu fer lo maestre de/la obra a-n Antoni ferrer una cana de ferre per pendre mesures de l'obra costa... .V. s".

${ }^{2}$ Felipe II (1527-1598) en las Cortes de Monzón (1585). Capitulo 89, unifica criterios métricos. Los procuradores remiten de la reducción de la cana de Tortosa a Barcelona. AHCTE, 387 (Comú II-63) Registre.
} 

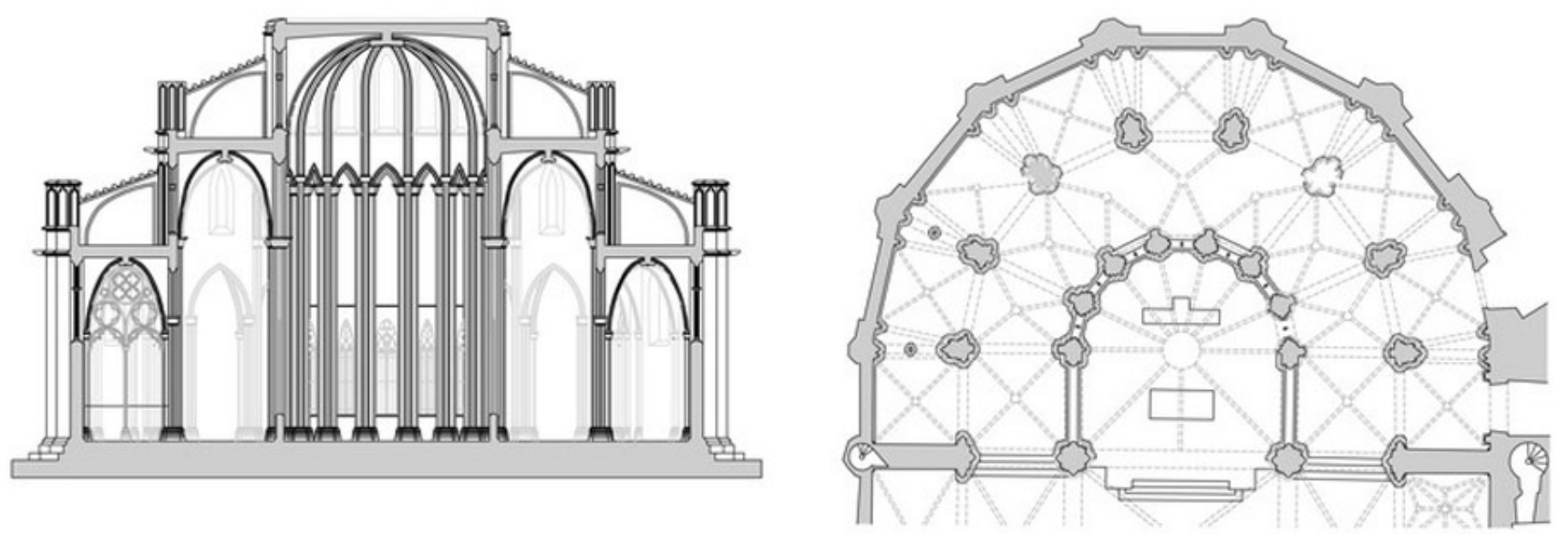

Figura 3. Sección y planta ábside catedral de Tortosa (1347-1441).

En la construcción de las capillas radiales se determinan tres momentos constructivos (Figura 4). El primero (1377-1383), con las pruebas iníciales del modelo, en las capillas de San Pedro, San Pablo y San Vicente. Después, entre (1387-1397), vendrá la consolidación y ajuste del modelo, con la construcción de las dos capillas siguientes. En el tercero, entre (14121424), se sistematiza la construcción de las cuatro capillas restantes (15). El nivel de cimbrado de las capillas radiales es claramente visible (Figura 5), y se dispuso a una altura variable, con la configuración que se muestra en la (Figura 6), y con la clave de la crucería situada a 45 palmos $(10,45 \mathrm{~m})$. La sección inicial de Tortosa tiene una proporción de (9/5). El maestro que cierra el primer cinturón del ábside, había construido las nueve capillas radiales de 3 canas de ancho, llegando a la última capilla radial exactamente a 150 palmos de diámetro. El replanteo del último pilar, se realizó exactamente a 108 palmos del primero. Con la finalización de capillas radiales se inicia también la ejecución del pilar major (1428) «...començam de desfer lo bastiment de la cerqua e començam lo bastiment del pilar major darrera l'altar, $e$ forenhi III mestres de axa e hun fadri...» (ACTo, ll. o. 14271428, núm. 13, fol. 15r).

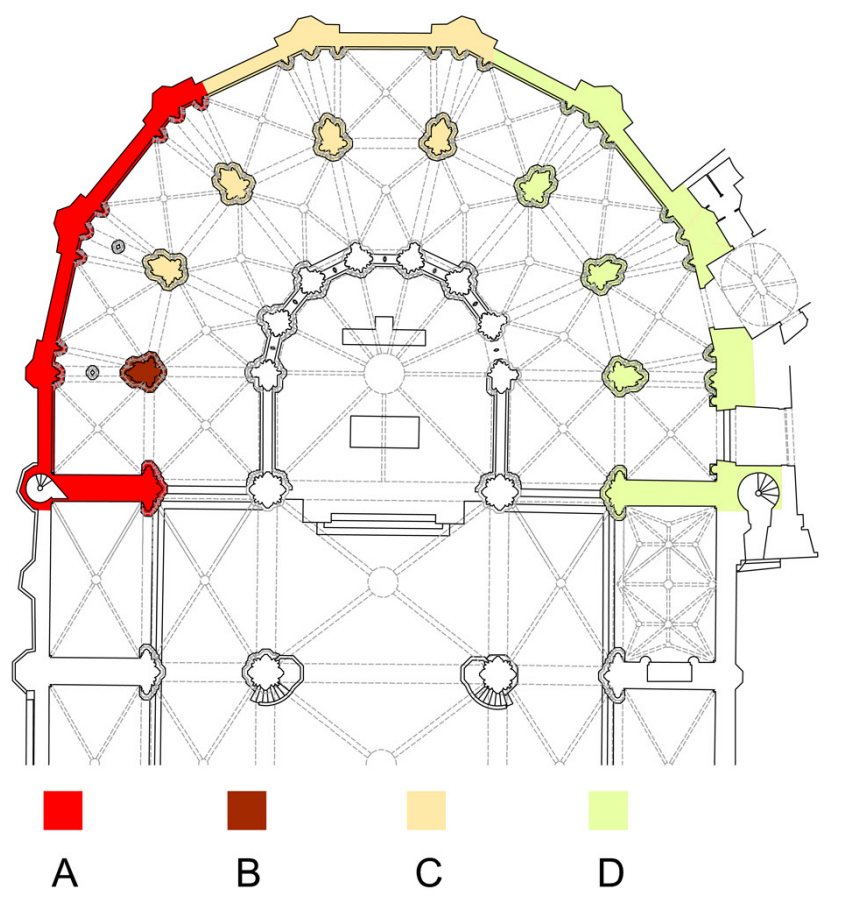

\section{UN MODELO NUEVO DE CATEDRAL, LA CONSTRUCCIÓN DE LA GIROLA}

La construcción de las bóvedas del deambulatorio se produjo entre (1424-1435). Fueron ejecutadas de forma simétrica sobre el eje central, a diferencia de las radiales que lo hicieron correlativamente (Figura 7). Estructuralmente se realizan cambios, ya que a diferencia de las radiales, los arcos formeros perpiaños y cruceros del deambulatorio se ejecutan sobre la misma imposta, a 11,67 m (5op+3d), y con el remate de la bóveda a 16,70 m (72p). En las capillas el despliegue de sección era (9/5), mientras que en la girola pasa a ser de (9/6). Tras el cierre de las capillas radiales, en mayo de 1428, se empieza construir el pilar major. Se trata de una acción coincidente con las obras de derribo en la cabecera de catedral románica, realizadas a partir del mes de agosto (16). El pilar se construye antes de la cerrar de las primeras bóvedas del deambulatorio realizadas en julio de 1431 y será desmontado en marzo de 1440, tras el descimbrado del presbiterio.

El maestro que traza la girola ha de construir los nuevos pilares del presbiterio, a la vez que el major. Desde el diseño

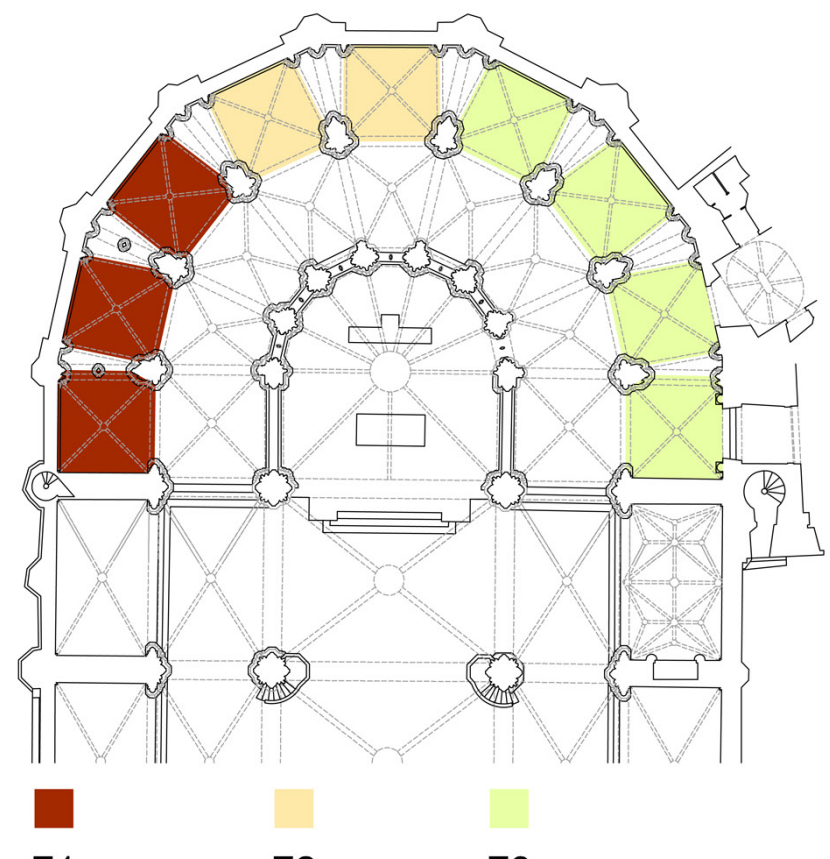

Figura 4. Evolución de magisterios A, B, C, D. Fases constructivas de las capillas radiales. 

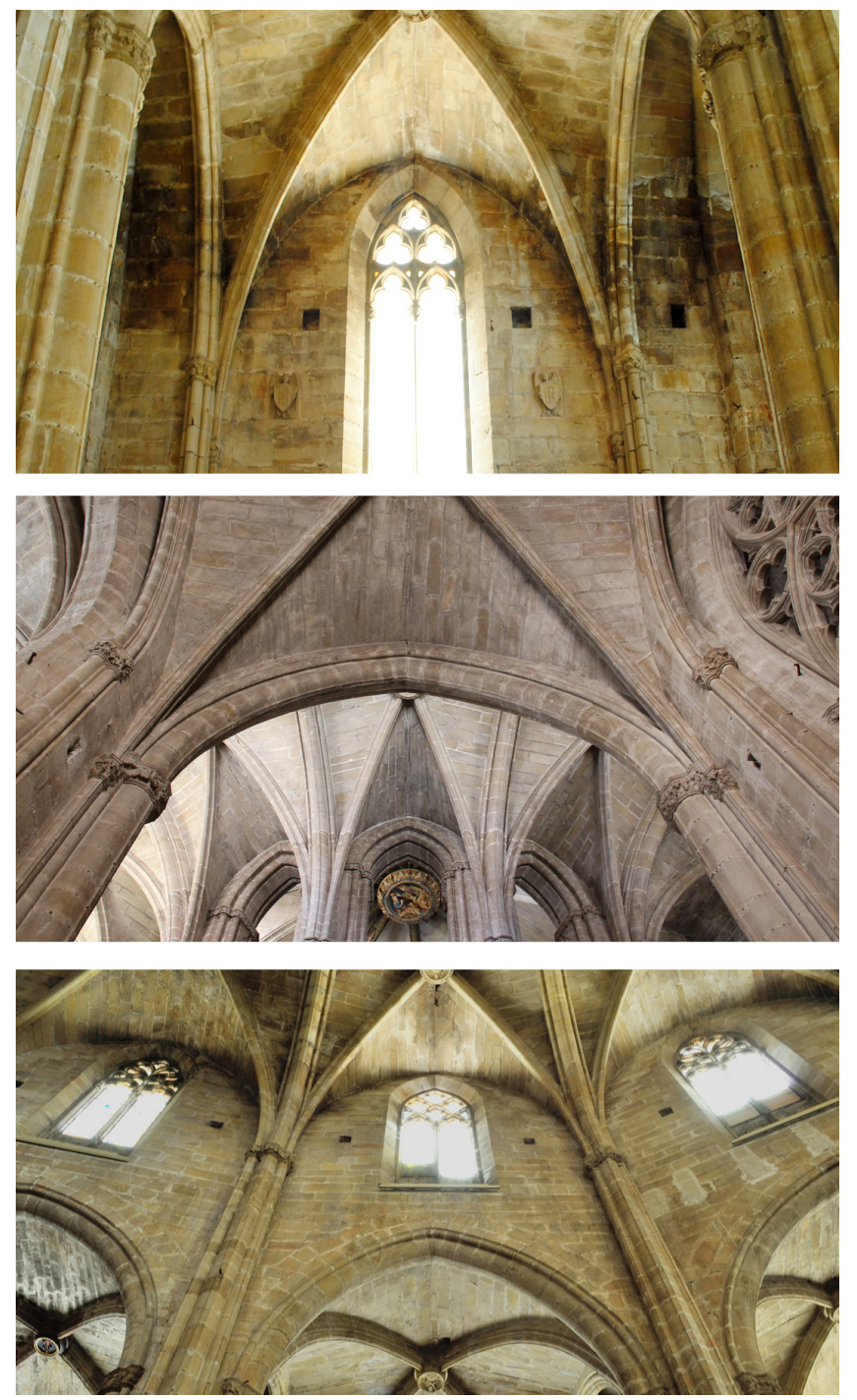

Figura 5. Improntas de sistema auxiliar en la Capilla de San Pedro (1383).

formal, tenia impuesta las molduras que concurrían desde las capillas radiales, pero podía proponer unas nuevas molduras en los arcos, que habían de formar las bóvedas del presbiterio. Los pilares disponen de dos modelos de molduras, en función de la concurrencia de los arcos (Figura 7); las iniciales sobre las capillas y las nuevas que convergerán en la clave del presbiterio, donde se sitúa el pilar major.

Los pilares de la boca del presbiterio, de sección similar a los del resto de la nave central, han de tener una sección suficiente como estribo y contrarresto. Cuestión bien diferente serán los ocho que cierran el presbiterio, ya que su diseño cambia significativamente. Los situados en el diámetro del presbiterio, ejecutados tras los de la nave, presentan una mayor dimensión en la dirección del eje longitudinal de la catedral.

Tras esta operación se empiezan a cerrar las bóvedas de la girola, primero las de planta cuadrada y luego las siete trapezoidales, situando el plano de cimbrado a unos 56 palmos. La estrategia constructiva de la girola es diferente a la de las capillas, así el deambulatorio se empieza a cubrir, simétrica- mente, por las capillas del evangelio y la epístola. Las bóvedas tienen un fácil contrarresto hacia el exterior de la fábrica, mediante los arbotantes de las capillas radiales que ya habían sido construidos. El equilibrio hacia el interior del presbiterio es algo más complicado, puesto que no dispone de más contrarresto que el pilar del presbiterio. Este hecho obliga a preguntarse qué ocurre con el empuje hacia el centro del presbiterio, antes de que éste fuera cubierto. La existencia de empujes hacia el interior y la construcción de las bóvedas de manera simétrica, hace pensar que pudieran ser reequilibrados por el pilar que se situó detrás del altar románico. El pilar major puede aparecer como elemento auxiliar, neutralizando estas acciones horizontales como un simple atirantado, que bien pudiera ser el andamiaje necesario para la construcción (Figuras 8, 9 y 10).

Las medidas estructurales del presbiterio arrojan una luz de $11,15 \mathrm{~m}$ (6 canas). El maestro fija la altura del remate del presbiterio a 100 palmos y traza la tirantez de arcos, bóvedas y el plano de cimbrado (17). En el caso de Tortosa, los puntos del cimbrado y del remate de la clave son conmensurables. Situados a la altura de la segunda terraza, son perfectamente visibles, aunque, a diferencia de las improntas restantes, éstas están tapiadas. Para determinar la curvatura de la arquería, se utiliza la perpendicular por el punto medio, desde el plano de cimbrado a la clave (18).

La primera operación para el cierre del presbiterio será la colocación de la clave con un peso aproximado de $8.746 \mathrm{Kp}$, que ha de situarse a 100 palmos. La talla de la clave ha de resolver, la labra de la iconografía de la Coronación de la Virgen María, y la geometría del corte de la cantería. La escultura inferior se dispone sobre una circunferencia de 10 palmos de diámetro, y el cuello de la clave ha de albergar la concurrencia geométrica de los nueve arcos cruceros. La medida de la clave esta implícita en la modulación de la base del pilar, y previamente diseñada y dimensionada en el momento que se traza la girola. Conociendo la relación (18/8) se puede determinar el radio de la circunferencia en función del nervio o viceversa (19). Los arcos cruceros del presbiterio tienen un ancho de 18 $\mathrm{cm}$, con lo que el cuello de la clave tiene $80 \mathrm{~cm}$, que son 3 palmos y medio, que corresponden exactamente a la proporción de Guarc (9/8) (20).

\section{LOS MÉTODOS GEOFÍSICOS Y LA TOPOGRAFÍA DEL PILAR MAJOR}

La campaña de Prospección geofísica (2012-2013)3, se realizó mediante un georadar modelo IDS Hi-Mod 200-60o MHz, con una resolución de lectura de 0.018Xo.2 m. El sistema ha permitiendo obtener visualizaciones alternativas de las zonas exploradas, con un mayor detalle (6oo $\mathrm{MHz}, 60$ nanosegundos) o una mayor profundidad (200 $\mathrm{MHz}, 90$ nanosegundos). La profundidad efectiva obtenida con los datos, oscila entre $1,8 \mathrm{~m}$. (60o MHz) y 2,3 m (200 MHz), calculando a una velocidad de 0,095 $\mathrm{m} /$ nanoseg (21).

La ubicación de la catedral románica planteaba dos hipótesis plausibles. El canónigo Matamoros (1932), la disponía perpendicular al eje actual, con orientación Norte-Sur, y próxima a la fachada principal (22), mientras que Victoria Almuni

\footnotetext{
Campaña realizada conjuntamente con, Roger Sala, Ekhine Garcia, Robert Tamba. Sala, R. (2013). Memòria d'Intervenció Prospecció geofísica a la catedral de Tortosa. SOT Prospecció Arqueològica.
} 

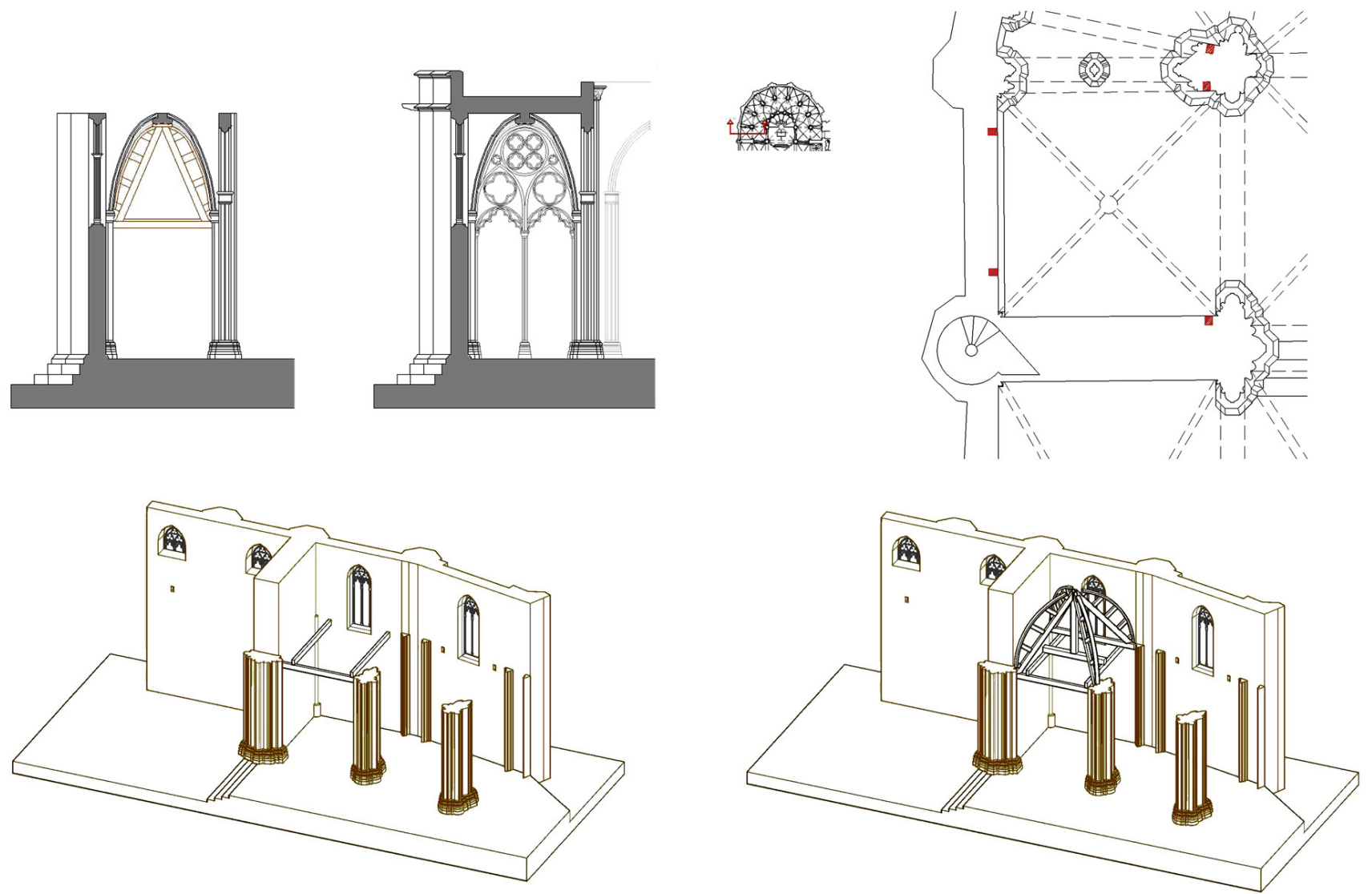

Figura 6. Hipótesis cimbrado Capilla de San Pedro (1383).
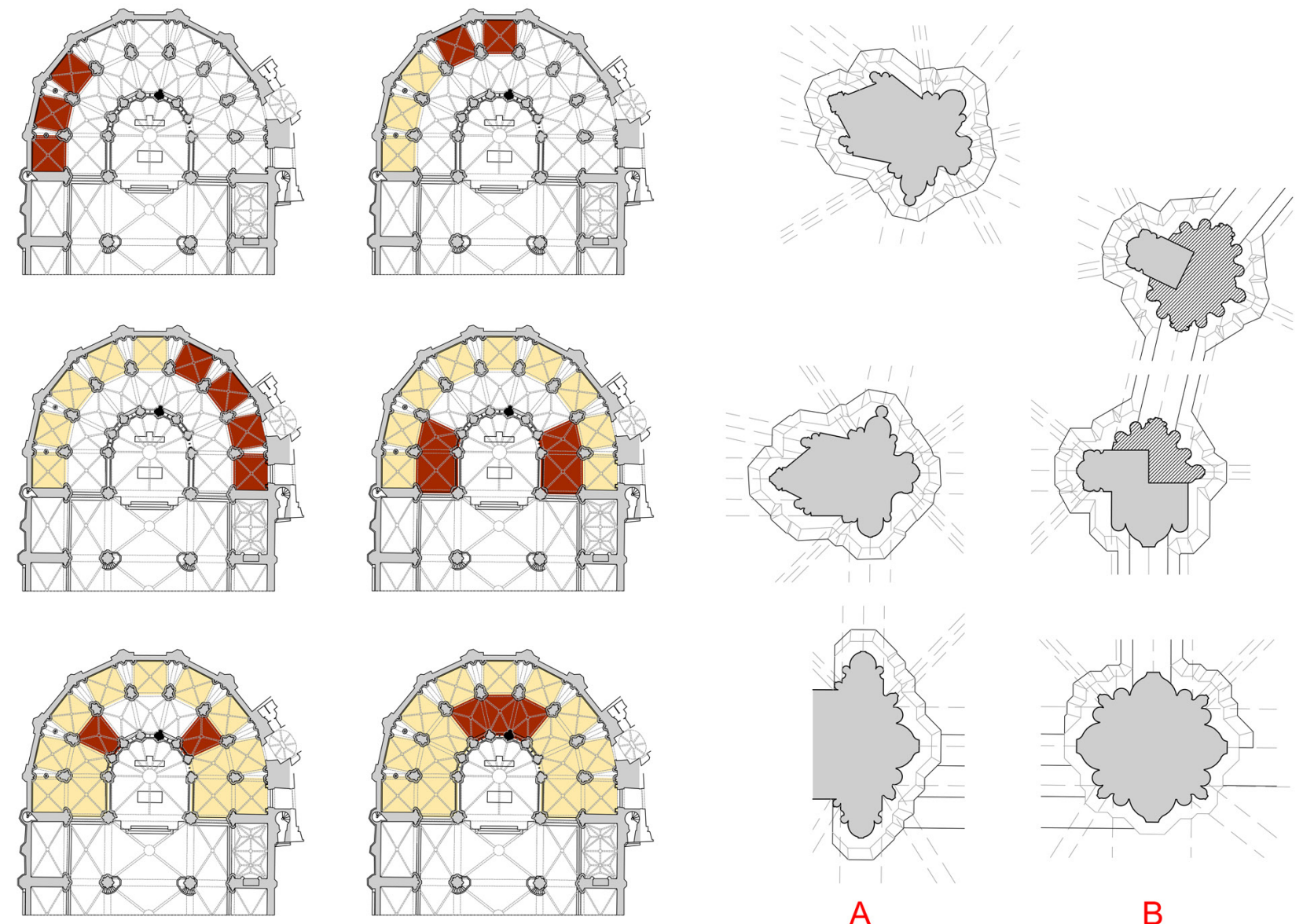

Figura 7. Evolución constructiva capillas radiales y girola (1383-1434). Tipología pilares y molduras girola; (A) capillas radiales, (B) presbiterio. 

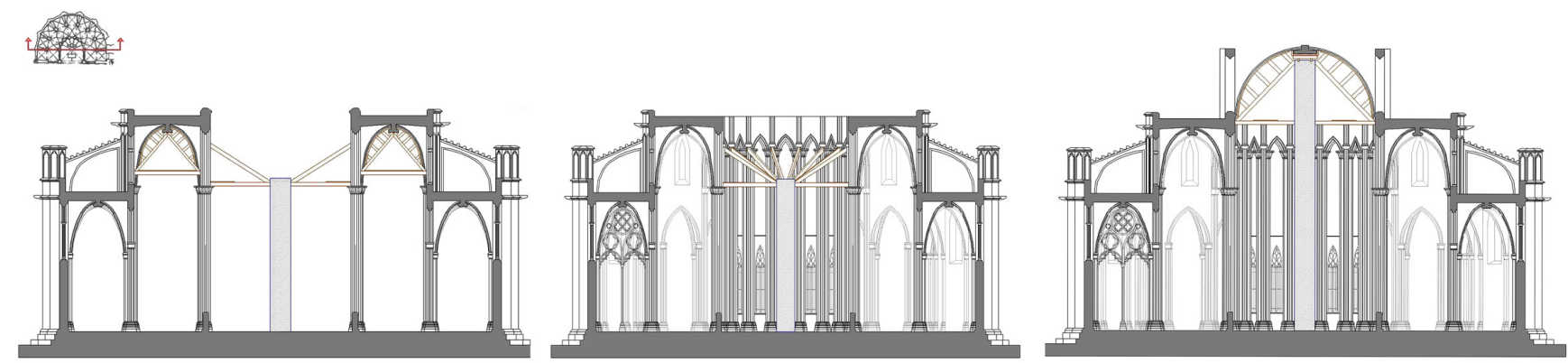

Figura 8. Hipótesis cimbrado deambulatorio y presbiterio.

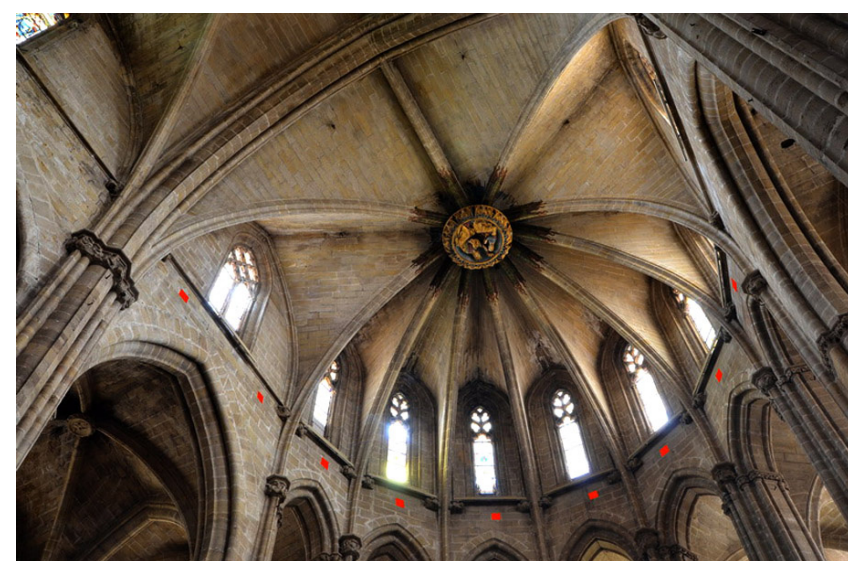

Figura 9. Improntas de los andamios en el presbiterio (1439-1440).

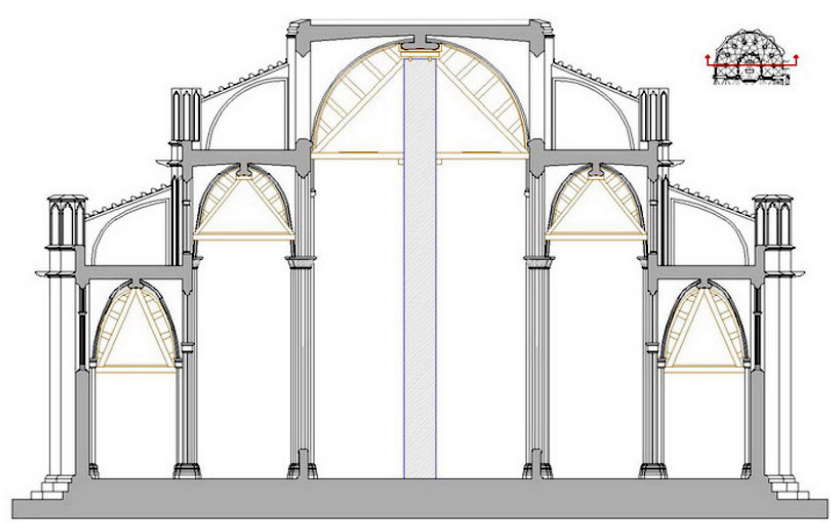

Figura 10. Hipótesis del cimbrado en la catedral de Tortosa (1383-1440).

(2004), la sitúa en una disposición similar a la catedral actual, orientada de Este-Oeste (16), ubicada al pie del presbiterio y apoyada sobre el claustro (23) (Figura 11). Los resultados obtenidos en el georadar (2012-13), muestran los elementos importantes de la catedral románica, con una orientación Este-Oeste, centrada sobre el eje de la catedral gótica, y con una cabecera concéntrica al presbiterio actual, diferente a las dos hipótesis iniciales.

Entre los datos obtenidos, en el corte de la sección (o,42 m 0,60 m s.s. e $600 \mathrm{MHz}$ ), se han encontrado tres áreas de respuesta convulsa. Entre ellas la anomalía-1 (Figura 12), que se identifica con un perímetro de alta amplitud, y centro de baja respuesta, detectada desde $0,3 \mathrm{~m}$ bajo la superficie perdiendo definición a $1 \mathrm{~m}$ de profundidad. El resultado se interpreta como un posible elemento constructivo colmatado.

Este elemento de forma circular, con una magnitud semejante a los pilares del presbiterio, esta situado en el centro del

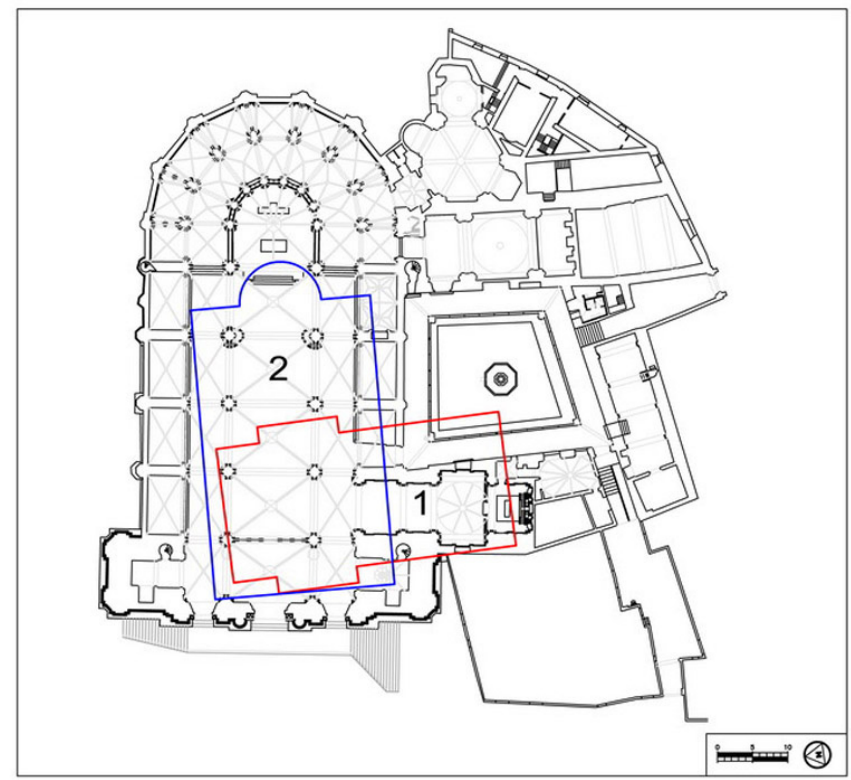

Figura 11. Hipótesis catedral románica sobre la gótica. 1. Matamoros, 2. Almuni.

altar, lo podemos relacionar con el pilar major. El pilar se situó, como se observa en el georadar (2012-13), tras el altar románico, tal vez en el cambio de la bóveda de cañón a la de cuarto de esfera. El carácter eventual se demuestra por su posterior desmontaje, en marzo de 1440 (ACTo, ll. o. 14391441, fol. 62r). A la vez dispone de una escasa cimentación, como indica el corte D del georadar (2102-13) (Figura 13). La operación del desmontaje del pilar major, se realiza tras el descimbrado del presbiterio, construyéndose, tras ello, el pavimento del sector, pasando a consagrar la catedral gótica (1441).

\section{LA POLIFUNCIONALIDAD COMO ELEMENTO AUXILIAR DEL PILAR MAJOR}

La estructura del ábside requiere la participación de todos sus elementos constructivos para formar un conjunto estable. El sistema de contrarresto es fundamental, y debe soportar los empujes de arcos y bóvedas una vez se ha cerrado el conjunto. Durante la construcción, la estabilidad en las fases intermedias se ha de conseguir mediante la utilización de elementos auxiliares.

Se ha realizado el estudio de la estática de la fábrica en el momento previo al cierre del presbiterio. El trazado de las líneas de presiones se realiza en el marco teórico del análisis límite (24) (25), según el cual podemos considerar que un arco será estable si es posible encontrar una línea de presiones contenida dentro de su sección. 
Constructivamente, la fábrica de los elementos verticales consta de muros y pilares macizos de piedra arenisca (densidad: $23 \mathrm{kN} / \mathrm{m}^{3}$ ). La luz se salva con bóvedas ojivales de piedra, considerando un espesor medio de $23 \mathrm{~cm}$ (un palmo). Sobre éstas, la cubierta se completa con un relleno de material cerámico aligerado y un importante grueso de mortero de cal (entre 40 y $50 \mathrm{~cm}$ ), rematado con la actual cubierta, con un peso total de $9 \mathrm{kN} / \mathrm{m}^{2}$. Los datos se han obtenido de las catas realizadas durante los trabajos del Plan Director (2000) (26). De esta manera, a partir de una hipótesis estable del ábside, se ha comprobado que efectivamente es imprescindible disponer de algún elemento auxiliar que contrarreste los empujes de las bóvedas (Figura 14).
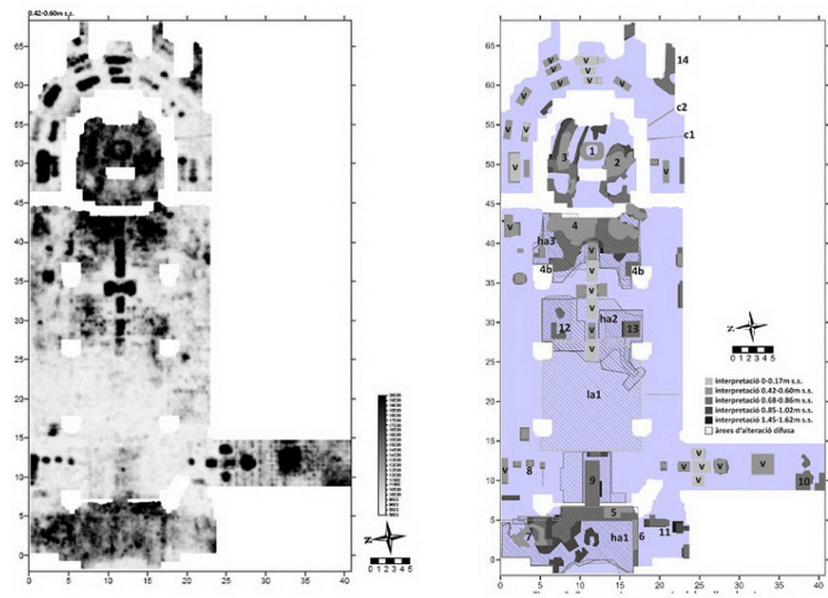

Figura 12. Imagen y anomalías en el corte de $0,42 \mathrm{~m}$ a $0,60 \mathrm{~m}$ 600 MHz. Georradar (2012-2013).
Según la hipótesis de cimbrado expuesta anteriormente se dispondría un puntal en cada pilar que transmitiría un empuje de unas $50 \mathrm{kN}$ al pilar major para compensar el empuje de la bóveda y los arcos (Figura 15). Los diferentes empujes horizontales se equilibrarían en la cabeza del pilar, obteniendo una solución estable del conjunto.

A demás de equilibrar los empujes de la girola, el pilar tendrá un papel fundamental en la colocación de la clave que preside el presbiterio. Ésta hubo de proyectarse tras la finalización de las capillas radiales, ya que se visualiza tanto desde la nave, como desde el doble deambulatorio. En este momento se desmontó la cabecera de la catedral románica, situada en el centro del presbiterio. Se determina además, la sección definitiva de la catedral; 150 palmos de ancho por 100 de altura (9/6). El diseño de los arcos del presbiterio esta ajustado a un ancho de seis palmos, para provocar una visión de la clave, tangente a su curvatura (Figura 16). De esta manera la gran clave preside visualmente el espacio y no solo con la visión frontal de la nave mayor, sino que también desde el deambulatorio.

La clave mayor es la primera pieza del cierre del presbiterio. En la documentación aparecen dos términos de medios auxiliares para realizar la operación del calado de la clave en su lugar, el bastiment y el matraç (2) ${ }^{4}$. Éste mismo término aparece en los libros de fábrica de la catedral de Barcelona (1418), en relación a la clave de la volta major de la façana (27). La operación final se realiza por fases, primero los marineros subieron la clave en una primera operación hasta un primer estadio, fins l'endret on devia muntar, para luego colocarlo con mas precisión, donde la clau avia a esser posada $e$ asetiada. El artefacto es acarreado mediante exarcia, especie de red gruesa. La clave con la delicada escultura, debía
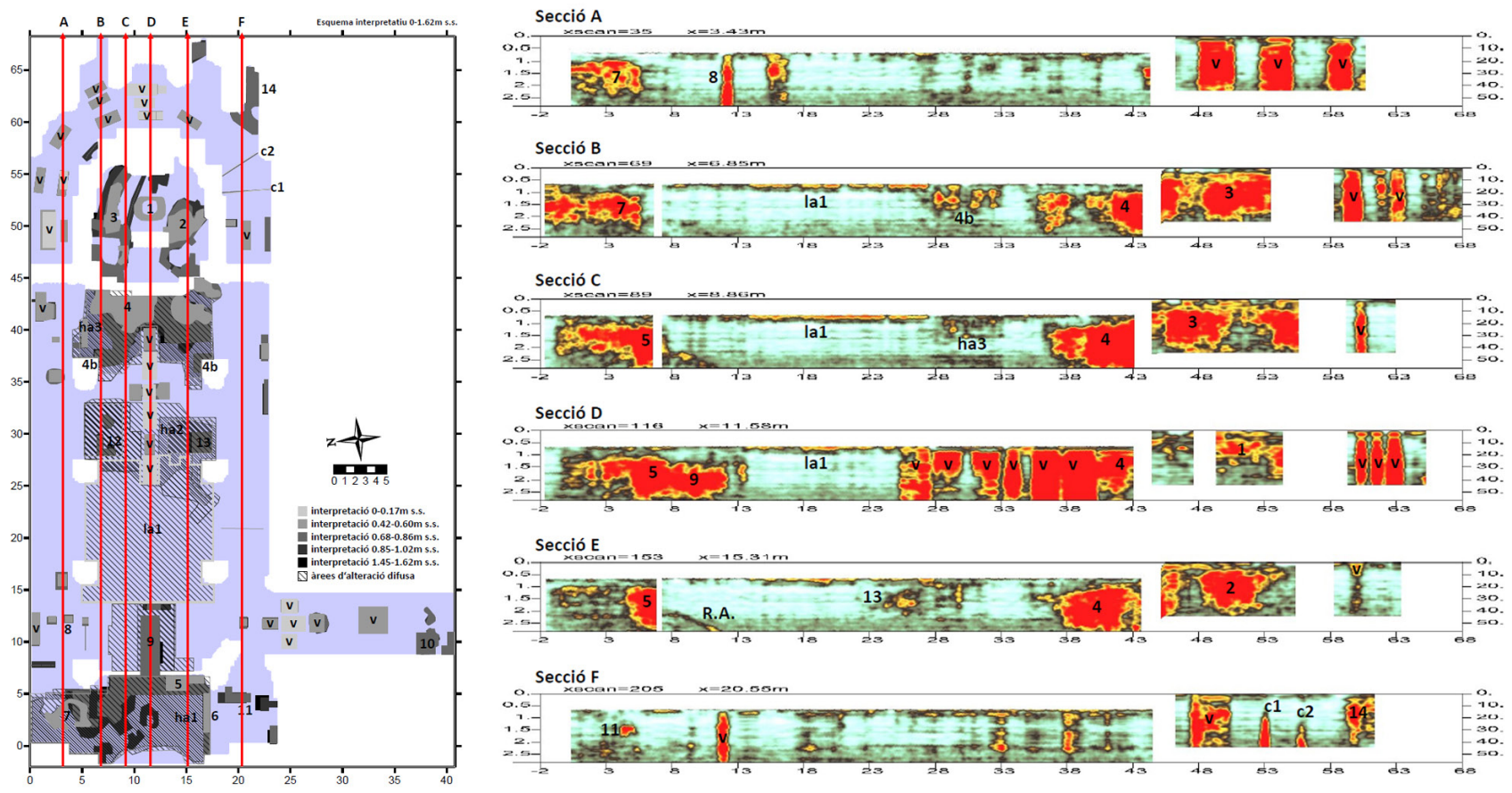

Secció C
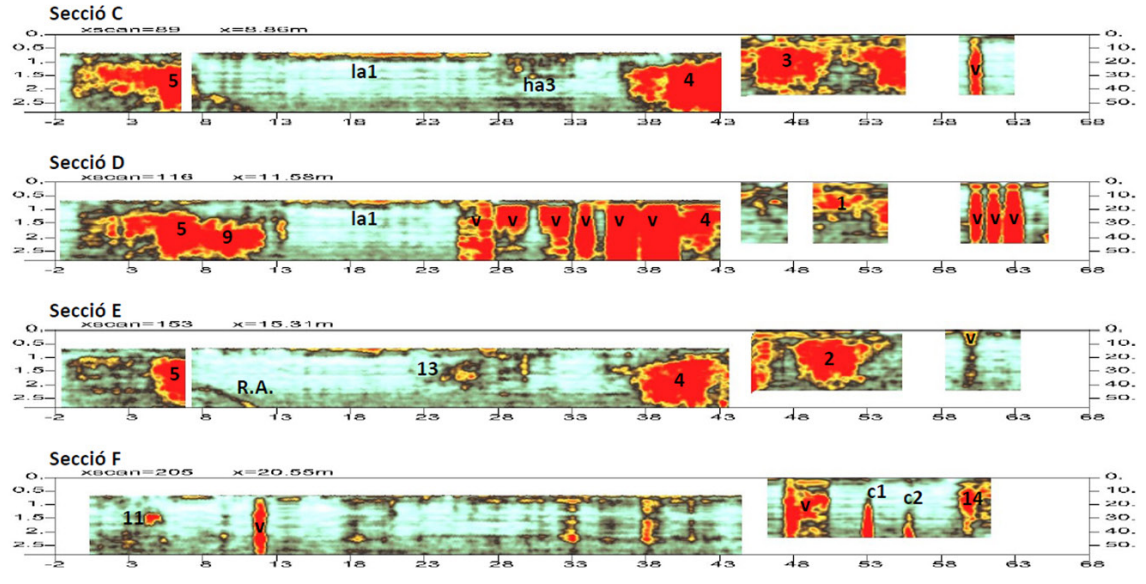

Figura 13. Anomalías y secciones del georadar (2012-2013).

\footnotetext{
${ }^{4}$ E essent muntada, muntaren e assentaren lo bastiment e matràs hon la dita clau avia a esser posada e asetiada, lo qual bastiment e matras asetiat davall la dita clau, ladonchs calaren la dita clau, e calant aquella per lo molt...la dita clau fonch damunt lo dit bastiment, $o$ matrás, ab lo maestre de la seu, assetiada, ...
} 
A1

A2

A3

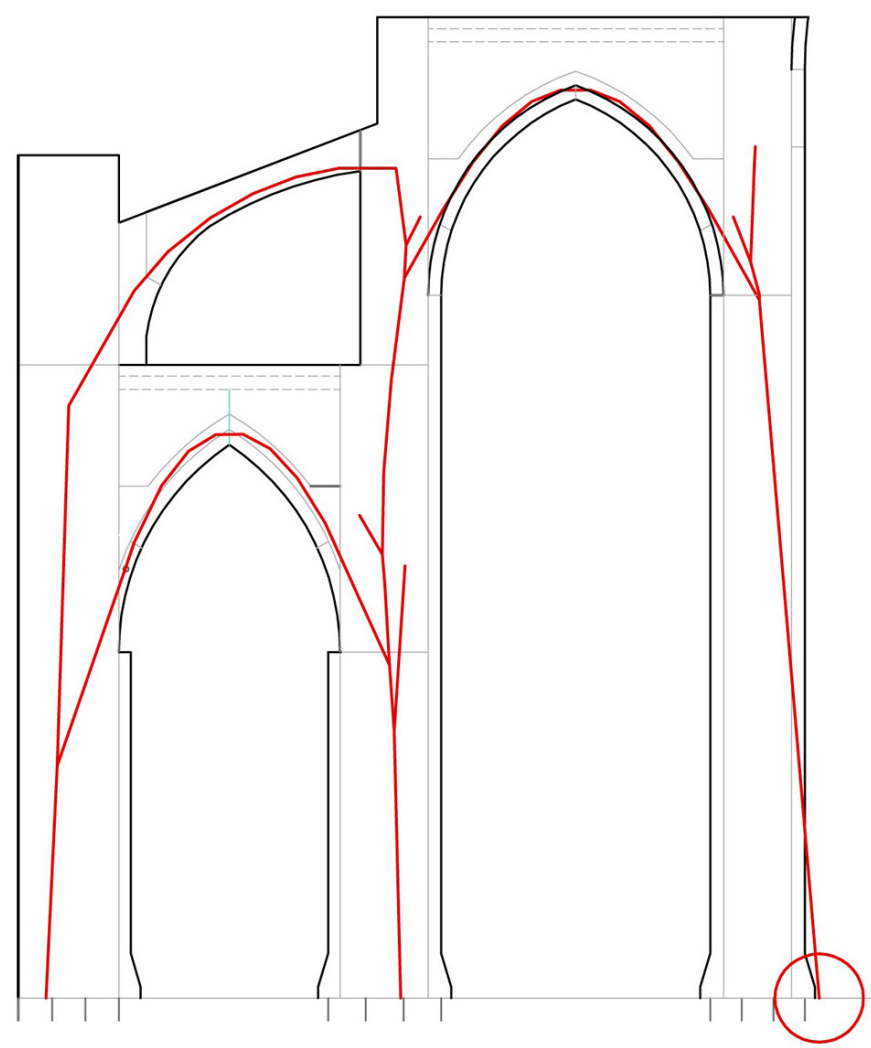

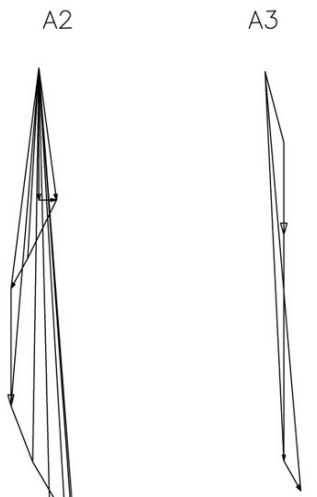

Figura 14. Línea de presiones capillas y deambulatorio. Hipótesis sin contrarresto.

A1

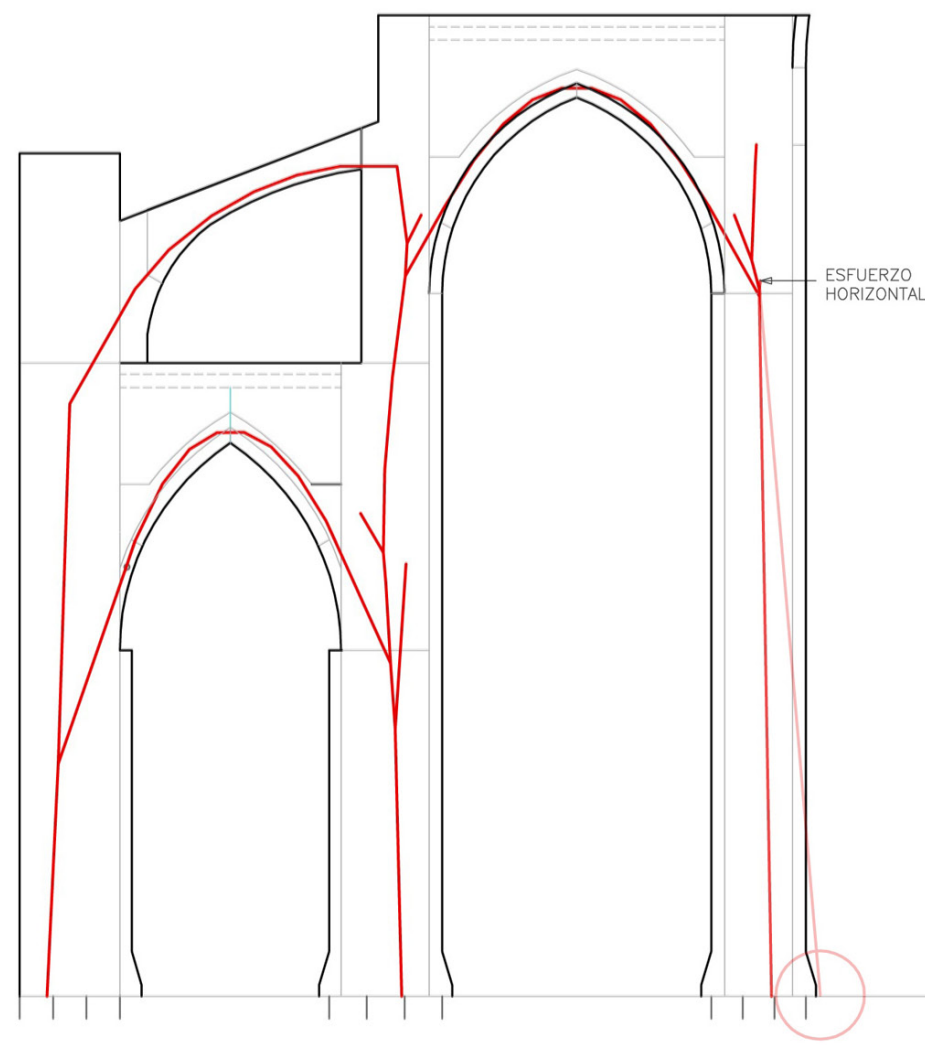

A1

A2

Figura 15. Línea de presiones capillas y deambulatorio. Hipótesis de contrarresto. 

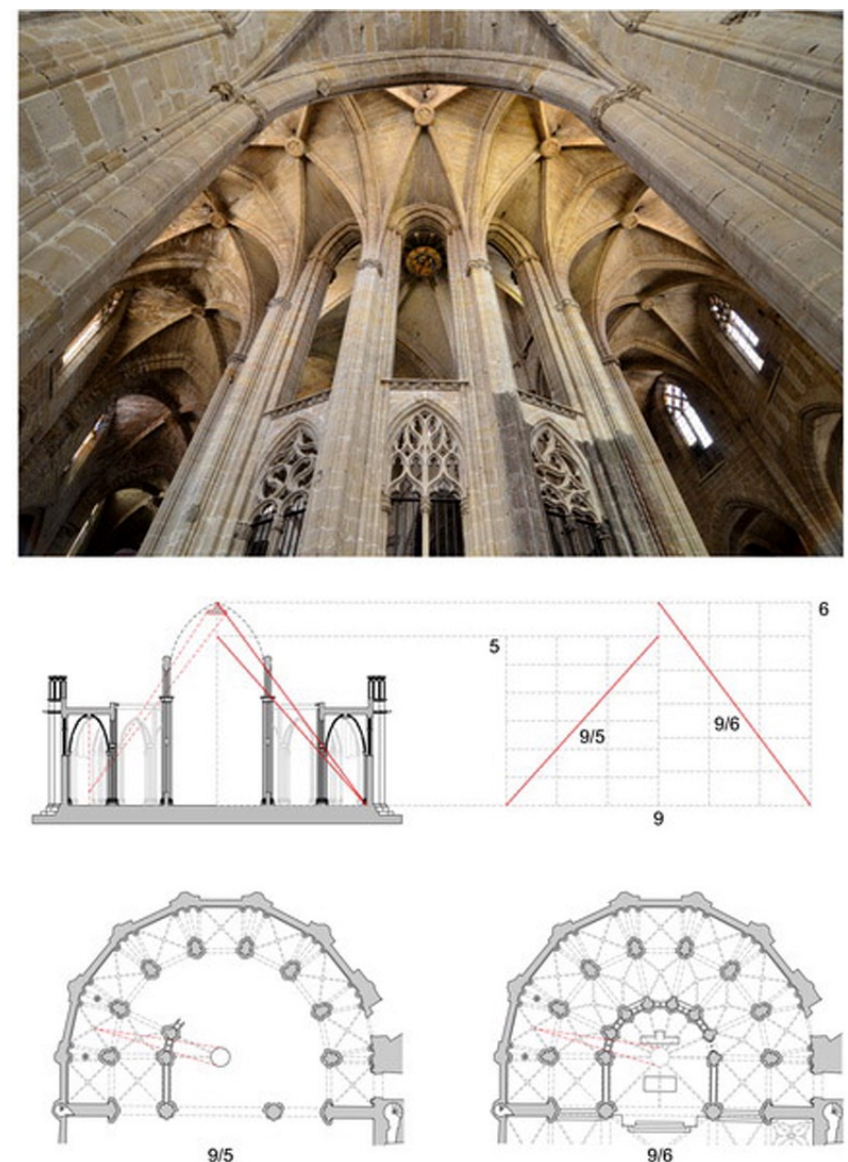

Figura 16. Cambio sección girola y fijación de la clave mayor a 100 palmos (1424).

estar protegida, y colocada sobre el bastiment, que ha de ser una especie de molde.

En el cielo solo existe la clave. La disposición geométrica y la orientación de ésta, ha de ser muy precisa, ya que han de converger los nueve arcos de las bóvedas. El bastiment podría disponer implícitamente la dirección de congruencia de los arcos. Pero este elemento, con la clave, ha de ser asetiada, sobre un elemento que contenga y recoja la geometría futura. Aquí es esencial la labor del pilar mayor, primero como sustento pero también como direccionador geométrico. Una hipótesis seria plantear otro bastiment, evidentemente de madera, sobre el pilar, en el que encajar el bastiment en la que se dispuso la clave. El bastiment inferior tendría además otra función, la de elemento de descimbrado. De esta manera al sacar las previsibles cuñas inferiores, se liberaría el conjunto de las bóvedas del presbiterio, permitiendo el asiento de la clave, con suficiente holgura para no dañar la base escultórica. Tras la colocación de la clave, en diciembre de 1439, se cimbra y se construye el arco de conexión entre el arco toral del presbiterio y la clave ${ }^{5}$. Se construyen las otras siete bóvedas concluyendo las operaciones en 1441.

\section{CONCLUSIONES}

En el gótico, la colocación de un pilar como elemento estructural central fue empleada en la construcción de estructuras de salas (28). Aparecerá en plancha XL de Villard de Honecourt (c.1175-1240) (29), con una estructura similar en la sala capitular de la catedral de Salisbury (1263-1284). Estas estructuras a manera de Palmier como se conoce en los Jacobins de Toulouse (1275-1292) (30), recogen hacia abajo, a manera de bóvedas de abanico, las estructuras de las bóvedas superiores (31). El pilar major que detectó el Georradar (2012-13) como la anomalía-1 es también un elemento central. Esta situado a tan solo $30 \mathrm{~cm}$ bajo la superficie actual, prácticamente en el centro del presbiterio, llegando a cerca de $1 \mathrm{~m}$ de profundidad. La anomalía-1 ha de ser el pilar major, elemento auxiliar, pero esencial para el sistema constructivo del ábside de la catedral de Tortosa. Este pilar auxiliar es un elemento polifuncional. En primer lugar como contrarresto en la construcción del deambulatorio. En segundo como estructura que facilita la colocación de la clave principal. Finalmente como soporte de andamiajes para el apeo del cierre del presbiterio.

\section{REFERENCIAS}

(1) Almuni-Balada, V. (2007). La catedral de Tortosa als segles del gòtic, vol 1., pp. 151-186. Barcelona: Fundació Noguera - Col-lecció Estudis.

(2) O'Callaghan, R. (1887). Anales de Tortosa I, pp. 17-20. Tortosa: Imprenta Católica de Gabriel Llasat.

(3) Lluis i Ginovart, J., Almuni-Balada, V. (2011, 26-29 de octubre). La clave de la clau. El cierre constructivo del presbiterio gótico. En Actas del Séptimo Congreso Nacional Historia de la Construcción, Santiago de Compostela, (pp. 733-743). Madrid: Instituto Juan de Herrera.

(4) Llunrat, A. (2013, 9-12 de octubre). Elementos y estructuras auxiliares en la construcción del ábside de la catedral de Tortosa. En Actas del Octavo Congreso Nacional de Historia de la construcción, Madrid, (pp. 537-544). Madrid: Instituto Juan de Herrera.

(5) García, S. (1990). Compendio de arquitectura y simetría de los templos conforme a la medida del cuerpo humano con algunas demostraciones de Geometria 1681 (Edición Facsimilar), pp. 65-66. Valladolid: COAV.

(6) Huerta, S., Ruiz, J. A. (2006). Some Notes on Gothic Building Processes: the Expertises of Segovia Cathedral. En II International Congress on Construction History, (pp. 1619-1632). London: Construction History Society.

(7) Huerta S. (2013). Technical Challenges in the Construction of Gothic Vaults: The Gothic Theory of Structural Design. En Bautechnik des historismus von den theorien vber gotische konstruktionen bis zu den baustellen des 19. Jahrhunderts. (pp. 163-195). Munchen: Ersc hienen im Hirm er Verlag.

(8) González-Varas, I. (2001). La razón constructiva: la Contención de la Ruina. En la Catedral de León, el sueño de la razón, (pp. 173-184). León: Edilesa.

\footnotetext{
${ }^{5}$ Item a XVII de dehembre feu serrar lo maestre un fust de un mul an Pere Bramon hon hac dos fi ls a rao de Is VI ds per obs del arch pla de la clau al arc major ...(ACTo, ll. o. 1439-1441, fols. 48r i 55r).
} 
(9) Villalobos, A. (2005, 27-29 de enero). Una aproximación al sistema de apeos de Adolfo Fernández Casanova para la catedral de Sevilla. En Huerta, S. (Ed.), Actas del Cuarto Congreso Nacional de Historia de la Construcción, Cádiz, (pp. 1091-1102). Madrid: I. Juan de Herrera, SEdHC, Arquitectos de Cádiz, COAAT Cádiz.

(10) Calma, J. M., Graciani, A. (2000, 26-28 de octubre). Sistemas de encimbrado y apeos en la Restauración Monumental española durante el siglo XIX. En Actas del Tercer Congreso Nacional de Historia de la Construcción, Sevilla, (pp. 153164). Madrid: Instituto Juan de Herrera.

(11) Dabas, M., Camerlynck, C., Freixas i Camps, P. (2000). Case History: Simultaneous use of electrostatic quadrupole and GPR in urban context: Investigation of the basement of the Cathedral de Girona (Catalunya, Spain). Geophysics, 65(2): 526-532.

(12) Macias, J. M., Muñoz, A., Teixell, I. (2012, julio-agosto). A la Recerca del Temple d'August, les intervencions arqueològiques a la catedral de Tarragona. Plecs d'Historia Local, 146: 2-4.

(13) Benito, M. A. (2013, 9-12 de octubre). Análisis detallado de estabilidad y sistema de contrarresto de la bóveda de la cabecera de la catedral del Salvador en Ávila. Influencia de la colocación de los arcos estribos del crucero en el siglo XVI. En Actas del Octavo Congreso Nacional de Historia de la construcción, Madrid, (pp. 103-112). Madrid: Instituto Juan de Herrera.

(14) Almuni, V. (1991). L'Obra de la Seu de Tortosa (1345-1441), p. 214. Tortosa: Cooperativa Gràfica Dertosense.

(15) Lluis i Ginovart, J. (2009, 21-24 de octubre). Evolución constructiva de los pilares de una girola gótica. El concepto de homogeneidad del material versus resistencia. En Actas del Sexto Congreso Nacional Historia de la Construcción, Valencia, (pp. 753-761). Madrid: Instituto Juan de Herrera.

(16) Almuni, V. (2004). La catedral románica de Tortosa. Aproximació documental a la seva historia. Recerca, 8: 211-250.

(17) Ungewitter, G., Mohrmann, K. (1890-1892). Lehrbuch der Gotischen Konstruktionen, Vol. 1, p. 118. Leipzig: Weigel Nachfolger.

(18) Willis, R. (1842). On the construction of the vaults of the middle ages. En Transactions of the Royal Institute of British Architects, Vol.1, part 2, p. 11. London: Longman.

(19) Lluis i Ginovart, J. (2008). La ciutat de Deu feta pels Homes. El cas de la catedral de Tortosa, pp. 17-41. Tortosa: Fundació fe y cultura Moceen Manya. Col-lecció: Llum Nova 2.

(20) Lluis i Ginovart, J., Fortuny, G., Costa-Jover, A., de Sola-Morales P. (2013). Gothic construction and the traça of a heptagonal apse: The problem of the heptagon. Nexus Network Journal, 15(2): 325-348, doi: http://dx.doi.org/ 10.1007/ so0004-013-0152-x.

(21) Sala, R. (2013). Memòria d'Intervenció Prospecció geofísica a la catedral de Tortosa. SOT Prospecció Arqueològica.

(22) Matamoros, J. (1932). La catedral de Tortosa. Trabajos monográficos acerca de su construcción y de su contenido artístico y religioso, pp. 8-9. Tortosa: Editorial Católica.

(23) Almuni-Balada, V. (2007). La catedral de Tortosa als segles del gòtic, vol. 1, pp. VI-IX. Barcelona: Fundació Noguera. Col-lecció Estudis.

(24) Heyman, J. (2005). El esqueleto de piedra. Madrid: Ed. Instituto Juan de Herrera.

(25) Huerta, S. (2005). Mecánica de las bóvedas de fábrica: el enfoque del equilibrio. Informes de la Construcción, 56(496): 73-89, doi: http://dx.doi.org/10.3989/ic.2005.v57.i496.496.

(26) Lluis, J., Llorca, A. (200o). Capítol Catedral de Tortosa. En Pla Director Sancta Maria Dertosae. Catedral de Tortosa (pp. 50). Tortosa: Generalitat de Catalunya.

(27) Carreras, F. (1914). Les obres de la catedral de Barcelona. Boletín de la Academia de Buenas Letras, 7(53): 302-317.

(28) Viollet-le-Duc, E. E. (1854-1868). Dictionnaire raisonné de l'architecture française du XIe au XVIe siècle, vol. 8 , p. 95. París: Bance et Morel.

(29) Lassus, J.B.A. (1858). Album de Villard de Honnecourt. Architecte du XIIIe siècle, (pp. 161-162). Paris: Imprimerie impériale.

(30) Sundt, R. A. (1989, junio). The Jacobin Church of Toulouse and the Origin of Its Double-Nave Plan. The Art Bulletin, 71(2): 185-207, doi: http://dx.doi.org/10.1080/00043079.1989.10788494.

(31) Carrasco, J. (2002). La estructura gótica catalana: Sobre los conceptos de medida y espacio. El problema de la forma en la cubierta (Tesis Doctoral), pp. 85-97. Barcelona: Departamento Expresión Gráfica Arquitectónica I - Universidad Politécnica de Catalunya. 